\title{
Féeries
}

Études sur le conte merveilleux, XVII $-\mathrm{XIX}{ }^{\mathrm{e}}$ siècle

$11 \mid 2014$

L'illustration des contes

\section{Le conte de fées français dans les planches populaires néerlandaises : trois versions du « Petit Chaperon rouge » (1781-1911)}

The French Fairy Tale in the Netherland's Popular Boards: Three Versions of

"Little Red Riding Hood" (1781-1911)

\section{Daphne M. Hoogenboezem}

\section{OpenEdition \\ Journals}

Édition électronique

URL : http://journals.openedition.org/feeries/941

DOI : 10.4000/feeries.941

ISSN : 1957-7753

\section{Éditeur}

UGA Éditions/Université Grenoble Alpes

\section{Édition imprimée}

Date de publication : 19 décembre 2014

Pagination : 125-145

ISBN : 978-2-84310-281-3

ISSN : $1766-2842$

\section{Référence électronique}

Daphne M. Hoogenboezem, « Le conte de fées français dans les planches populaires néerlandaises : trois versions du « Petit Chaperon rouge » (1781-1911) », Féeries [En ligne], 11 | 2014, mis en ligne le 19 décembre 2015, consulté le 29 octobre 2020. URL : http://journals.openedition.org/feeries/941 ; DOI : https://doi.org/10.4000/feeries.941 


\section{LE CONTE DE FÉES FRANÇAIS DANS LES PLANCHES POPULAIRES NÉERLANDAISES : TROIS VERSIONS DU «PETIT CHAPERON ROUGE» (1781-1911)} salons littéraires parisiens. Or une définition claire et généralement acceptée du nouveau genre fait alors défaut. Un débat intertextuel sur la définition commence ${ }^{\mathrm{I}}$. Les visions des auteurs se dessinent à travers un jeu de références dans lequel outre le texte, les illustrations vont jouer un rôle important ${ }^{2}$. Si les conteuses, dont Madame d'Aulnoy, Mademoiselle de La Force, Mademoiselle L'Héritier et Madame de Murat, placent leurs contes dans une tradition écrite ancienne ${ }^{3}$ et en soulignent le caractère littéraire ${ }^{4}$, une vision plus folklorique du genre commence à se former dans les recueils de Charles Perrault. Le frontispice figurant la vieille conteuse paysanne entourée de son jeune public en constitue le résumé visuel. Cette image, réutilisée dans d'innombrables rééditions françaises et étrangères, s'est alors gravée dans l'esprit du public. À la fin du

I. Voir S. Raynard, «Perrault et les conteuses précieuses de la génération I690: Dialogue ou querelle masquée», The Romanic Review, vol. 3, n 4, 2008, p. 317-331.

2. Pour une analyse de ce débat intertextuel, qui se poursuit d'ailleurs dans les éditions anglaises et néerlandaises des contes de Perrault et de Madame d'Aulnoy, voir D. M. Hoogenboezem, Le conte de fées en images. Le rôle de l'illustration chez Perrault et Madame d'Aulnoy (I695-1800), 2012.

3. Les conteuses établissent un lien entre leurs contes et une tradition littéraire ancienne écrite remontant au Moyen Âge. Ainsi, Mademoiselle de La Force indique, dans les Contes des contes, qu'elle a pris le conte "dans un ancien livre gothique, nommé Perseval» (Mademoiselle de La Force, "Avis pour le conte suivant» dans Contes des contes, Paris, S. Bernard, I697, p. 75). Dans ses Euvres meslées, Mademoiselle L'Héritier soutient que les contes populaires proviennent des « romans» des troubadours (Mademoiselle L'Héritier et al., Contes, R. Robert, Paris, H. Champion, 2005, p. 36). Madame de Murat précise, dans l'avertissement qui précède ses Histoires sublimes et allégoriques, qu'elle s'est inspirée d'un recueil de nouvelles écrites par l'auteur italien de la Renaissance, Giovan Francesco Straparola. Voir D. M. Hoogenboezem, ouvr. cité, p. II.

4. Leurs contes sont plus longs et plus complexes que ceux de Charles Perrault. Ils sont écrits dans un style précieux, et comportent de nombreuses références à la mythologie et aux ouvrages littéraires de l'époque, tels que les Fables de Jean de La Fontaine ou les romans de Mademoiselle de Scudéry. 
$\mathrm{XVIII}^{\mathrm{e}}$ siècle, la conception des contes de fées comme des histoires populaires pour enfants transmises oralement depuis un temps immémorial n'est plus guère remise en question. L'illustration reste cependant un élément important de la plupart des éditions, celles pour enfants notamment, mais elle se dote d'une nouvelle fonction esthétique et didactique. Elle offre désormais des informations sur un autre sujet : l'évolution des idées pédagogiques. Le présent article offrira un exemple de cette seconde fonction de l'illustration.

L'illustration est également un élément essentiel dans les planches populaires. En effet, ces planches, des feuilles de 30 x $40 \mathrm{~cm}$ environ dont seul le recto est imprimé, comportent en général une ou plusieurs images tandis que le texte est le plus souvent réduit à une simple légende. Aux Pays-Bas, des planches paraissent à partir du $\mathrm{Xv}^{\mathrm{e}}$ siècle. Elles deviennent extrêmement populaires, entre autres grâce à la disponibilité de papier bon marché, à l'absence de censure ou d'ingérence des pouvoirs publics et à leur prix abordable. Pour réduire les frais, elles sont produits en grosses quantités, souvent avec du papier de mauvaise qualité et des planches de bois usées ou vermoulues, et coloriées sans soin avec des pochoirs ou simplement avec le pouce (coloriées «à la manière hollandaise», suivant l'expression consacrée). Elles sont ensuite vendues par des libraires ou des colporteurs. Leurs thèmes sont extrêmement variés. On trouve des planches religieuses résumant des histoires bibliques ou des vies de saints, d'autres portant sur les mois ou les saisons, les fêtes populaires (la Saint-Martin, la Saint-Nicolas), les bonnes manières, les proverbes, les métiers ou les jeux d'enfants ; des adaptations de textes littéraires tels que Don Quichotte, Gulliver ou Robinson Crusoé. On trouve également des planches sur des thèmes fantastiques tels que le monde à l'envers, le pays de Cocagne, ou des personnages folkloriques tels que Till l'Espiègle et Jan de Wasser. Elles s'adressent à un public très vaste, riche et pauvre, jeune et adulte. À la fin du XVII ${ }^{\mathrm{e}}$ siècle paraissent les premières planches qui s'adressent de façon explicite aux enfants ( $d e$ jonge jeugd»), pourtant la différence entre les planches populaires et les planches pour enfants est encore souvent difficile à percevoir jusqu'à la fin du XVIII ${ }^{\mathrm{e}}$ siècles.

5. Pour informations sur les planches populaires et enfantines, voir J. Salman, «Kinderprenten», dans M. van Delft (éd.), Wonderland. De wereld van het kinderboek, Zwolle, Waanders, 2003, p. IO-II et A. Borms, Centsprenten: de cartoons van weleer, dans Origine, tijdschrift voor kunst, antiek en toegepaste kunst, vol. I4, ${ }^{\circ}$ 2, 2006, p. 50-56. 
Si les premières éditions des contes de fées français sont publiées aux Pays-Bas dès la fin du Xvir siècle sous forme livresque, leurs éditions sous forme de planches paraissent relativement tard et constituent une part modeste du répertoire des planches populaires. Les premières sont des éditions pirates qui reproduisent (avec plus ou moins de fautes) le texte français, souvent accompagnées de reproductions des illustrations figurant dans les éditions originales. Ainsi les premières éditions néerlandaises des contes de Perrault paraissent à Amsterdam chez Adriaan Moetjens (I694I697) et Jaques Desbordes (I697 et I698). La publication en I754, par Pierre van Os, d'une première édition bilingue des contes de Perrault marque une étape importante dans l'évolution du recueil. Comme l'indique son titre 6 , il s'adresse explicitement à la jeunesse et a un but didactique : l'apprentissage du français par les enfants néerlandais. Enfin paraissent dans les années $\mathrm{I} 77 \mathrm{O}$ et $\mathrm{I} 78 \mathrm{O}$ les premières éditions comportant seulement le texte néerlandais. Dès lors, les contes de Perrault appartiennent au domaine naissant de la littérature enfantine, et ils en deviennent immédiatement l'un des ouvrages les plus prisés. Ce n'est qu'à partir de ce moment qu'ils sont également adaptés dans des planches populaires. Leurs éditeurs ontils voulu profiter de cette deuxième vogue des contes de Perrault? Les premières planches représentant des contes ne s'adressent pas de façon explicite aux enfants, mais l'époque de publication, le choix dominant de contes convenant particulièrement à ces derniers, de par leurs jeunes héros et leur contenu moralisant ${ }^{7}$, sont les principales caractéristiques de ces adaptations suggérant que les éditeurs visaient essentiellement un public jeune.

L'adaptation des contes dans des planches implique une diversification importante de leur iconographie. Dans les planches populaires, chaque conte est illustré de quatre à vingt images. Jusqu'alors un conte n'avait été illustré que d'une ou deux seulement. Comme l'a montré Carine Picaud, il se crée autour des contes de Perrault une imagerie restreinte :

La Belle au Bois dormant est représentée endormie, le prince à son chevet; Le Petit Poucet tire les bottes de l'ogre ; la Barbe bleue courroucé s'apprête à décapiter sa trop curieuse épouse dont les frères arrivent au galop. Les scènes retenues pour illustrer

6. "Contes de ma Mère l'Oye - Vertellingen van Moeder de Gans, met negen keurlyke koopere plaatjes, zeer dienstig voor de jeugdt om haarzelve in het Fransch en Hollands te oefenen" ("Contes de ma Mère l'Oye, avec neuf gravures sur cuivre excellentes, très utiles à la jeunesse pour s'exercer au français et au hollandais»), La Haye, Pierre van Os, I754.

7. Le plus souvent nous ne disposons pas de listes des planches publiées par les différents éditeurs, cependant les planches ayant survécu à ce jour donnent un indice de leur répertoire. Des contes de Perrault, «Le Petit Chaperon rouge» et «Le Petit Poucet», semblent avoir été publiés le plus souvent dans des planches. 
les autres contes représentent davantage de variantes : l'illustration de "Cendrillon» oscille entre la fuite du bal et l'essayage de la pantoufle ; «Le Petit Chaperon rouge» est tantôt illustré par la dévoration de la fillette, tantôt par la rencontre du loup en chemin, cette dernière scène, moins crue, supplantant progressivement la première ; l'imagerie du "Chat botté» alterne la discussion avec les moissonneurs, la noyade simulée et l'entrée de la princesse au bras du marquis suivi du chat dans le château de l'ogre ${ }^{8}$.

Dans les planches on retrouve souvent ces mêmes scènes, mais également de nombreuses autres images. Le texte, en revanche, est réduit à une simple légende de trois ou quatre lignes au plus. L'image va donc permettre de créer une nouvelle lecture des contes, d'introduire des variantes et parfois un peu plus de suspense dans ces histoires fixées à l'écrit depuis un siècle, et souvent antérieurement connues du public.

Dans l'étude qui suit nous analyserons trois planches populaires néerlandaises du conte "Le Petit Chaperon rouge" " publiées entre 1780 et I9II ${ }^{\mathrm{IO}}$. La première planche, publiée par Erve H. Rynders au début des années I780, est l'une des plus anciennes ayant ce conte pour sujet. De plus, elle présente une version qui a été très largement répandue parce qu'elle a été rééditée plusieurs fois par la suite. Les deux autres planches, publiées par Dirk Noothoven van Goor et Brepols \& Dierckx zoon à la seconde moitié du $\mathrm{XIX}^{\mathrm{e}}$ siècle, offrent deux versions très différentes du conte. Ces différences semblent refléter les débats pédagogiques des XVIII ${ }^{\mathrm{e}}$ et $\mathrm{XIX}^{\mathrm{e}}$ siècles. Dans notre analyse nous tiendrons compte de plusieurs aspects des planches : le nombre d'images et leur disposition, le sens de la lecture, la technique d'illustration, la composition des images, et enfin le choix des scènes. L'importance que l'adaptateur accorde aux différentes parties de l'histoire offre des informations sur les éléments qu'il a voulu souligner ou, au contraire, réduire ou supprimer. On peut parfois en déduire les idées pédagogiques qui sous-tendent ces réalisations. Comme la mise

8. C. Picaud, «L'illustration du conte de fées. Enjeux d'images, visions d'imagiers», dans O. Piffault (éd.), Il était une fois... les contes des fées, 200I, p. I56.

9. Si la version du conte «Le Petit Chaperon rouge» des frères Grimm est connue aux Pays-Bas dès le début du XIX ${ }^{e}$ siècle, les planches sont le plus souvent basées sur la version de Perrault. La fin du conte n'est pas adaptée. Comme l’explique J. Bos («De sprookjes van Grimm», dans M. van Delft (éd.), Wonderland. p. 32-33), une première traduction néerlandaise de vingt contes des Grimm paraît dès I820, mais le recueil n'a d'abord que peu de succès. Ce n'est qu'à la seconde moitié du XIX ${ }^{e}$ siècle que de nouvelles éditions néerlandaises des contes des Grimm commencent à paraître. C'est peut-être pour cette raison que les éditeurs des planches préferent se baser sur la version de Perrault.

Io. La date estimée de publication des planches est basée sur les années d'activité des éditeurs. 
en images du conte implique une certaine théâtralisation du récit, nous utiliserons la terminologie du théâtre, et notamment les termes désignant les moments de l'action dramatique (exposition, intrigue, climax et catastrophe/dénouement ${ }^{\mathrm{II}}$ ), afin de déterminer l'importance accordée aux différentes parties de l'histoire.

\section{De vertelling van Roodkapje, Amsterdam, Erve H. Rynders (Gerard Oortman), I78I-I854}

La première planche (fig. I), qui semble avoir été créée vers I78I, se compose de douze illustrations : ce sont des gravures sur bois assez rustiques entourées de cadres doubles et réparties en quatre bandes (A-D) de trois images. Chaque illustration est accompagnée d'une légende en néerlandais d'une à trois lignes. On constate donc que, comme dans la plupart des planches, une «lecture» linéaire de l'histoire est induite par cette disposition. Cependant les vignettes ne se succèdent pas simplement. Leur organisation horizontale en quatre bandes découpe le récit de sorte que chacune corresponde à l'une des quatre parties de l'histoire, créant ainsi une version étonnamment harmonieuse du conte. Ainsi les trois premières images $\left(\mathrm{AI}_{\mathrm{I}}-\mathrm{A}_{3}\right)$ correspondent à l'exposition. Le créateur de la planche introduit le Petit Chaperon rouge, sa mère et sa grand-mère. On remarque que la mission de la jeune héroïne et l'interdiction de lambiner en chemin sont formulées de façon explicite. L'intrigue occupe les trois illustrations suivantes (BI-B3). Dans la forêt, le Chaperon fait la rencontre du loup, qui l'aborde. À l'arrière-plan on aperçoit des bûcherons qui travaillent dans la forêt, mais qui ne sont pas mentionnés dans le texte. C'est l'une des rares fois où les illustrations de cette planche ajoutent des informations non fournies par le texte. La planche pourrait ainsi servir de point de départ à un conteur qui voudrait, à partir d'elle, ajouter d'autres élé-

II. L'exposition permet d'introduire les personnages du conte et de décrire la mission de l'héroïne. L'intrigue présente le problème central du conte : le Chaperon rencontre le loup et lui révèle où elle va. Au climax la tension s'accroît : le Chaperon s'amuse à cueillir des fleurs tandis que le loup se précipite chez la grand-mère et la dévore. La catastrophe et le dénouement racontent respectivement l'événement qui mène à la chute de l'héroïne (le Chaperon entre dans la maison et se met au lit) et la fin du conte (la dévoration de la jeune fille). Ces deux derniers actes sont si étroitement liés dans le conte que nous les considérons comme une seule partie du récit. I. Nières a constaté une structure comparable dans une planche d'Épinal figurant le conte «La Barbe Bleue» dont elle fait l'analyse dans "Une planche d'Épinal : Histoire de la Barbe Bleue : NE IIO2", J. Perrot (éd.), Tricentenaire Charles Perrault — Les grands contes du XVII e siècle et leur fortune littéraire, 1998, p. 75-84. 
ments à cette version du conte. Or, en général, la légende correspond très exactement à la scène représentée dans l'image. Il s'agit peut-être d'un premier indice du public visé par l'adaptateur : les images peuvent servir à un enfant d'aide à la lecture. La troisième bande de la planche (CI$\left.\mathrm{C}_{3}\right)$ résume le climax : on voit la jeune hérö̈ne courir après les papillons alors qu'en arrière-plan le loup est déjà devant la porte de la maison de la grand-mère $\left(\mathrm{CI}_{1}\right)$. Ensuite il dévore la grand-mère $\left(\mathrm{C}_{2}\right)$ et le Chaperon arrive $\left(\mathrm{C}_{3}\right)$. La catastrophe et le dénouement occupent les trois dernières illustrations $\left(\mathrm{D}_{1}-\mathrm{D}_{3}\right)$. La jeune fille pose ses vêtements sur la chaise et se couche dans le lit. Le dialogue final est réduit à une seule question : «Ma mère-grand, que vous avez de grandes dents!» ("Grootmoeder, wat hebt gy groote tanden!», D2). La réponse du loup "C'est pour te manger» ("Dat is, om $u$ des te beter te kunnen opeten") coïncide avec la dévoration de la jeune fille représentée dans la dernière image $\left(\mathrm{D}_{3}\right)$.

Cette première planche offre une version assez fidèle et équilibrée du conte. Le créateur semble avoir voulu transmettre une leçon morale: comme la mission du Chaperon est formulée très explicitement au début de l'histoire, le lecteur doit conclure que la jeune fille a commis une erreur en s'amusant à courir après les papillons au lieu d'aller directement chez sa grand-mère. Les aspects violents et sexuels de l'histoire sont présents mais sans en être les éléments dominants. Les deux dévorations, celle de la grand-mère et celle du Chaperon, ont été représentées mais, dans la plupart des autres illustrations représentant à la fois le loup et la jeune fille, la tension dramatique est atténuée par une présence humaine (B2 : des bûcherons ; $\mathrm{B}_{3}$ : un moulin à l'horizon ; $\mathrm{C}_{\mathrm{I}}$ : des maisons), ou par une certaine ouverture de l'image suggérant que l'héroïne peut encore échapper au loup. En outre, le créateur limite les références sexuelles dans l'histoire. S’il représente le Chaperon et le loup au lit, dans cette image la position de ce dernier, couché sous les draps et à peine visible derrière l'héroïne, ne ressemble point à celle de la gravure d'Antoine Clouzier ornant l'édition originale de Perrault : le loup y est à moitié couché sur la jeune fille, séducteur-violeur qu'il est ${ }^{\mathrm{t} 2}$.

Cette planche a souvent été réimprimée. On en trouve au moins cinq versions. Les gravures sont différentes, mais les scènes représentées et leur

I2. Voir A. Gaillard, Fables, Mythes, Contes. L'esthétique de la fable et du fabuleux (I660-I729), 1996, p. 254. 
disposition sur la feuille sont identiques ${ }^{13}$. Comme l'indiquent les longs (sous-)titres de certaines de ces rééditions, ces planches s'adressent de façon explicite à un public jeune, exprimant clairement leur visée moralisante. Ainsi nous lisons sur la planche publiée par T. C. Hoffers : «Veuillez joindre à la prudence, Ô Jeunesse! également la sagesse. Ainsi les deux vous épargneront beaucoup de chagrin" ("Wil bij voorzichtigheid, O Jeugd, ook wijsheid paren: zoo zal die beide u voor veel verdriet bewaren»), et sur celle de J. Noman : «Le Petit Chaperon rouge, les enfants, que vous connaissez, vous est représenté dans cette planche d'après le conte de l'Oye». Ces paratextes dévoilent le public visé et le but moral des planches, mais également la stratégie commerciale des éditeurs : ils soulignent l'importance des illustrations, moins nombreuses dans les livres de contes, et renvoient en même temps au recueil de Perrault, connu par les enfants néerlandais sous le titre de "Contes de ma Mère l'Oye" ("Sprookjes van Moeder de Gans»). Il était, dès la seconde moitié du XviII ${ }^{\mathrm{e}}$ siècle, l'un des titres les plus prisés de la littérature enfantine. S'il est encore souvent difficile de percevoir la différence entre les planches populaires et les planches à destination des enfants des $\mathrm{XVII}^{\mathrm{e}}$ et XVIII ${ }^{\mathrm{e}}$ siècles, la destination de celle-ci ne faisait aucun doute.

13. Roodkapje $N^{\circ}$ 38, Rotterdam, T. C. Hoffers, I820-I839; Histoire du Petit Chaperon rouge - Historie van Roodkapje, Schaarbeek, Hemeleers van Houter, I827-I894 ; Roodkapje, kind'ren! $U$ bekend, wordt, bij vernieuwing, thans $U$ voorgesteld in deze prent, naar het spreukje van de Gans. $N^{\circ} 4$ I, Zaltbommel, J. Noman \& zoon, Schalekamp en van de Grampel, I830-1850 Roodkapje, kind'ren! U bekend, wordt, bij vernieuwing, thans $U$ voorgesteld in deze prent, naar het spreukje van de Gans. No 206, Turnhout, Glenisson en van Gerechten, I833-1900 ; Roodkapje, kind'ren! U bekend, wordt, bij vernieuwing, thans $U$ voorgesteld in deze prent, naar het spreukje van de Gans. $N^{5} 30$, 's Hertogenbosch, Lutkie en Cranenburgh, I848-188I. 


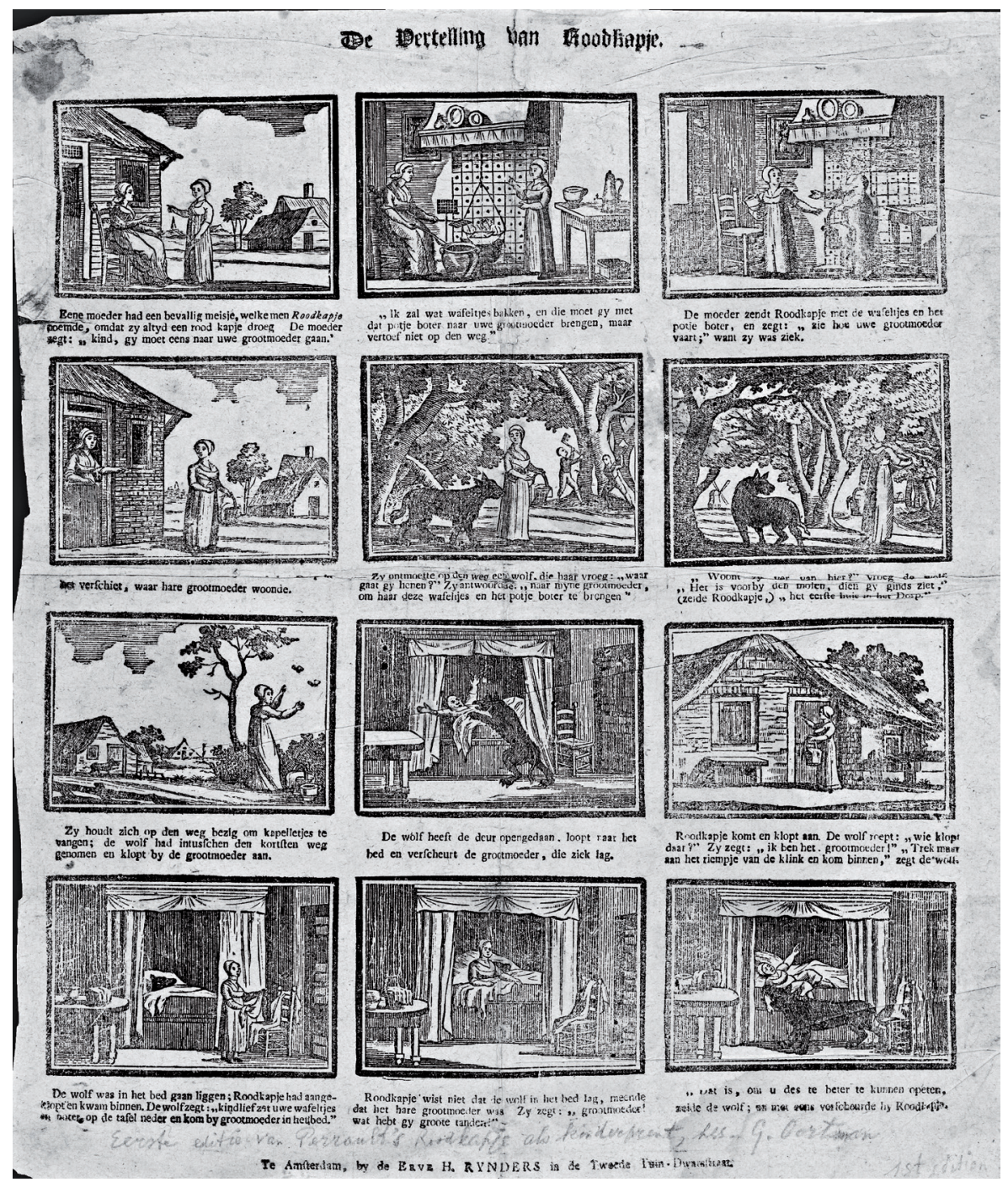

Fig I. - De vertelling van Roodkapje, Amsterdam, Erve H. Rynders (Gerard Oortman), I78I-1854. I2 gravures sur bois, 37 x $32 \mathrm{~cm}$. Bibliothèque royale (KB), La Haye : SMC KoIz2. 


\section{De Geschiedenis van Roodkapje, No 204, Leiden, D. Noothoven van Goor, I850-1880}

Dans la seconde planche (fig. 2), Dirk Noothoven van Goor, un éditeur qui publie de nombreux livres et planches pour enfants, crée une version du conte dans laquelle les aspects violents et sexuels ont presque entièrement disparu. Cet effet est dû au choix des scènes et à leur disposition sur la feuille, mais aussi aux modifications du sens de la lecture et de la technique d'illustration. La planche se compose de quatorze illustrations. Chaque image est accompagnée d'une légende : un petit poème de quatre vers en néerlandais. Elles sont réparties en trois colonnes (I à III) de quatre ou cinq images. Ces colonnes se lisent de haut en bas. Le lecteur doit donc alors remonter du bas jusqu'en haut de la page pour pouvoir poursuivre sa lecture. Noothoven emploie des lithographies au lieu des gravures sur bois de fil, ce qui permet de réaliser des illustrations plus détaillées et réalistes. De plus, les cadres de ces dernières ont disparu. Comme nous le verrons plus bas, ces deux changements dans l'emploi du médium ont une influence importante sur la lecture de la planche.

Nous commencerons par commenter le choix des scènes. Si dans la planche précédente l'adaptateur accordait trois illustrations à chacune des quatre parties de l'histoire, la répartition est moins équilibrée dans la seconde planche. Noothoven met l'accent sur la première partie de l'histoire. L'exposition est plus élaborée (quatre illustrations sur quatorze). Le créateur de la planche donne plus d'informations sur le chaperon. Il ajoute une image détaillée du modèle et une description de l'étoffe — du velours rouge avec des dentelles noires - dans le texte. Il donne également des informations supplémentaires sur l'héroïne du conte. Il s'agit d'une fille de Gelderland, l'une des provinces des Pays-Bas (I, I). En outre, l'adaptateur accentue le caractère sage du Petit Chaperon rouge dès le début de l'histoire. Il la décrit comme aimée de tous à cause de ses nombreuses vertus $(\mathrm{I}, 2)$. Il donne ensuite un exemple de son comportement irréprochable : un jour elle aperçoit dans l'une des pièces de la maison un kouglof («een versch gebakken tulband») et un pot de beurre, mais puisqu' elle est une fille sage elle ne touche pas au gâteau $(\mathrm{I}, 3)$. Sa mère lui demande ensuite de l'apporter à sa grand-mère $(\mathrm{I}, 4)$. 


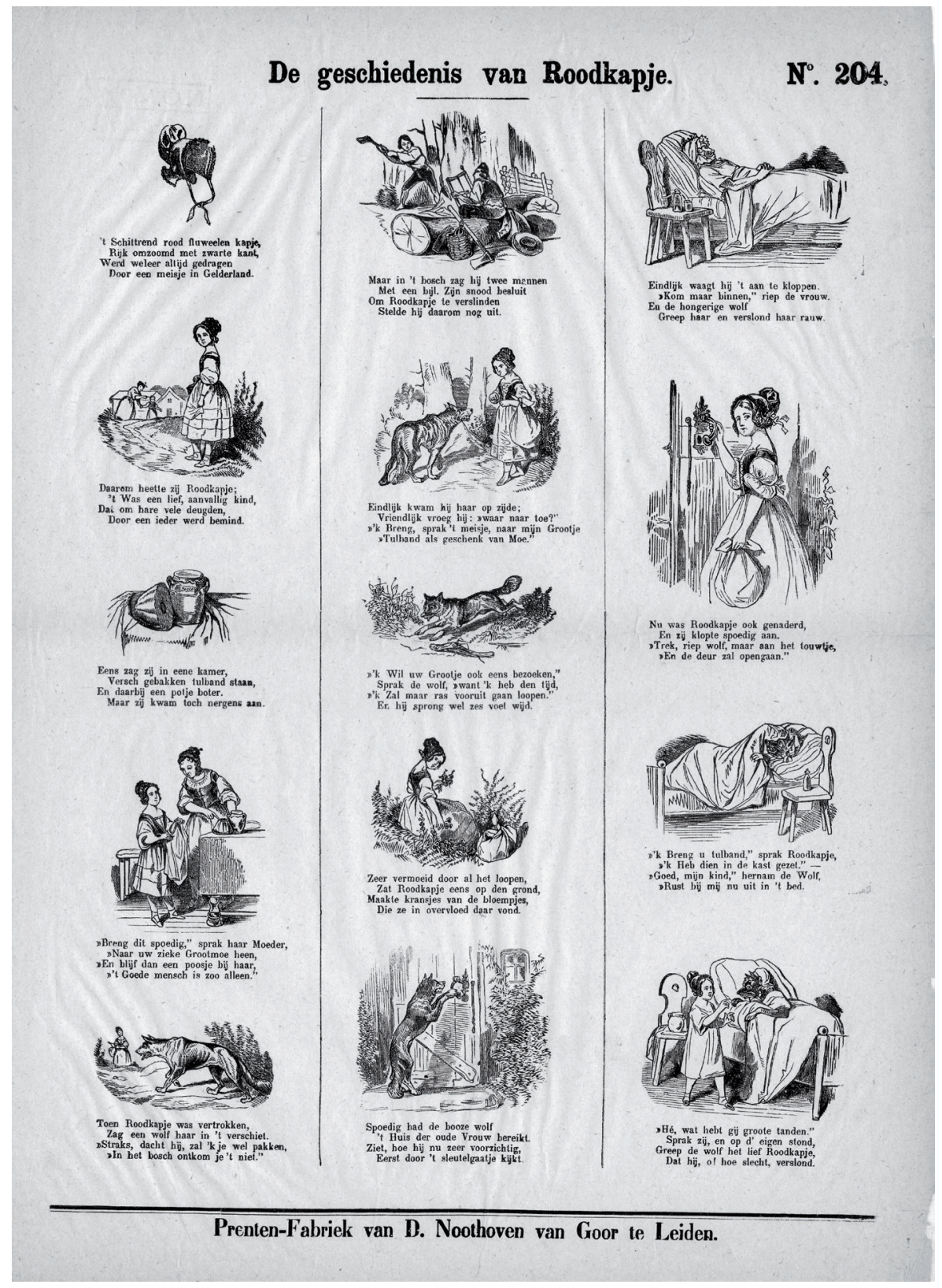

Fig. 2. - De Geschiedenis van Roodkapje. N. 204, Leiden, D. Noothoven van Goor, I850-1880. I4 lithographies, $42 \times 30 \mathrm{~cm}$.

Collection Nederland Openluchtmuseum, Arnhem : PR I024. 
L'intrigue occupe également quatre illustrations. Avant même que le Petit Chaperon rouge ne fasse la rencontre du loup, l'adaptateur avertit son jeune public : le loup veut manger l'héroïne (I, 5). Heureusement des bûcherons travaillent dans la forêt et le méchant loup se voit obligé de remettre à plus tard son projet (II, I). Il lui demande cependant où elle va (II, 2) et court ensuite vers la maison de la grand-mère (II, 3 ).

Les quatre illustrations suivantes donnent à voir le climax de l'histoire. L'adaptateur de la planche fournit un deuxième exemple de la bonté de la jeune fille. Celle-ci ne s'assoit qu'un petit moment parce qu'elle est fatiguée de la promenade, et elle tresse alors quelques guirlandes de petites fleurs qu'elle trouve autour d'elle. Il n'est plus question dans cette version d'une jeune fille désobéissante ou naïve qui perd son temps parce qu'elle préfère s'amuser (II, 4). Entre-temps le loup arrive chez la grand-mère (II, 5) et la dévore (III, I). Si le texte évoque brièvement cet événement cruel, l'illustration montre la scène qui la précède : la bonne vieille est couchée dans son lit, le loup n'est pas encore entré dans la maison.

Les images III, 3 et III, 4 correspondent à la catastrophe et au dénouement. On voit le loup couché dans le lit tandis que le Chaperon s'approche. Si la dévoration de la jeune fille est décrite dans la dernière légende, l'adaptateur a préféré encore une fois montrer une scène antérieure moins violente. En mettant ainsi l'accent sur la première partie de l'histoire (l'exposition, l'intrigue et le climax occupent douze des quatorze illustrations), il limite les aspects violents et sexuels du conte, mais ce n'est pas tout : il ne représente point les dévorations et il réduit le nombre d'illustrations dans lesquelles le Petit Chaperon rouge et le loup sont représentés ensemble. Il n'y en a que trois dans cette planche alors que dans la précédente il y en avait six. En outre, grâce à la composition des images, la distance physique entre ces deux personnages semble être plus grande. Le plus souvent ils se trouvent dans un espace ouvert. L'absence d'arbres et de buissons (I, 5), celle de baldaquin et de murs autour du lit (III, 3 et III, 4), donnent l'impression que l'héroïne peut encore échapper au loup. Cet effet est renforcé par le manque de cadres autour des illustrations, et par le fait que les espaces blancs entre elles sont relativement grands. Cela induit un nouveau parcours de lecture qui permet de ralentir quelque peu le rythme de l'histoire et de différer la fin cruelle du conte. Pour le lecteur occidental qui n'est point habitué à une telle mise en page, ce parcours original auquel invite la planche rappelle peut-être un chemin 
zigzagant à travers la forêt ${ }^{14}$. Enfin l'adaptateur réduit les connotations sexuelles : le Petit Chaperon rouge n'est pas représenté au lit avec le loup et l'aspect animalier de ce personnage est accentué dans le texte comme dans les illustrations. Le loup marche le plus souvent à quatre pattes, il porte rarement des habits et il "parle» relativement peu. Ainsi, dans la légende de l'image III, 4, au lieu de répondre à la question du Chaperon, il la dévore. Le loup "parle» uniquement dans les légendes des illustrations II, 2 et II, 3 et III, 2 et III, 3, alors que dans la planche d'Erve H. Rynders six légendes rapportent les paroles du loup $\left(\mathrm{B}_{2}, \mathrm{~B}_{3}, \mathrm{C}_{3}, \mathrm{D}_{1}, \mathrm{D}_{2}, \mathrm{D}_{3}\right)$.

La planche de Noothoven exprime une vision pédagogique qui trouve son origine au siècle des Lumières. Sous l'influence des philosophes, on commence à s'intéresser davantage à l'éducation des enfants. On souligne notamment l'importance de la raison. Aux Pays-Bas, les pédagogues illuminés Justus Van Effen (I684-I735) et Elizabeth Wolff-Bekker (I738-I804), appelée communément Betje Wolff, déconseillent les contes (populaires) comme lectures pour la jeunesse. Ils les considèrent comme des histoires irrationnelles et mensongères et incitent les parents à raconter plutôt des récits historiques authentiques à leurs enfants. Dans son périodique Le Misanthrope (I745), Van Effen range les inventeurs des contes parmi les menteurs ${ }^{15}$. Wolff écrit une étude sur l'éducation des enfants adressée aux mères néerlandaises intitulée Proeve over de opvoeding (1779), dans laquelle elle exprime son mépris pour les "contes imbéciles»:

Comment! Ne pourrait-on point dire à un enfant intelligent et attentif, à qui l'on peut raconter un conte imbécile, quelque chose qui mériterait une meilleure désignation? L'histoire ancienne ou moderne, nationale aussi bien qu'étrangère, ne fournit-elle donc rien qui soit adapté à la réceptivité d'un tel enfant? ${ }^{16}$

Au XIX ${ }^{\mathrm{e}}$ siècle, nombreux sont encore les partisans d'une telle vision. On crée même une compagnie chargée de l'éducation de la population : "Maatschappij tot het Nut van het Algemeen" ("Compagnie d'utilité géné-

I4. A. Renonciat décrit plusieurs images dans lesquelles le créateur a joué avec le sens de la lecture pour évoquer le parcours du protagoniste à travers la forêt. Voir "Le Petit Poucet en images", dans S. Le Men et A. Renonciat (éd.), Livres d'enfants, livres d'images (I848-I9I4), Les Dossiers du Musée d'Orsay n ${ }^{\circ}$ 35, 1989, p. 23-26 et «Petit Poucet dans la jonchée de feuilles", Le Vieux Papier, n 316 , I990, p. 205-218.

15. Le Misanthrope (tome 3), 1745, p. 282.

16. "Hoe! zou men een schrander en opmerkzaam kind, dat men een zot sprookje vertellen kan, niet iets kunnen zeggen dat eenen beteren naam verdiende? Leveren de oude en nieuwe geschiedenissen, en zowel de vaderlandsche als de uitheemsche, dan niets uit, geschikt naar de vatbaarheid van zo een kind? ", E. Wolff-Bekker, Proeve over de opvoeding aan de Nederlandse moeders, 1780, p. 57 [nous traduisons]. 
rale»). Cette compagnie lutte entre autres contre les planches populaires, qu'elle considère comme grossières et immorales. Het Nut souhaite améliorer le contenu et la valeur pédagogique des planches pour enfants et encourage les éditeurs à publier des planches éducatives. Leur offensive réussit, bien que des planches populaires naïves et non épurées continuent de paraître. Les nouvelles planches se caractérisent par leur langue soignée et leurs images irréprochables. Les textes deviennent plus informatifs mais également plus normatifs et moralisateurs. Les thèmes des nouvelles images pour enfants sont souvent didactiques : ils ont trait à l'histoire nationale, l'emploi des outils ou la zoologie. Parfois le contenu des planches traditionnelles est adapté. Les contes de fées ne disparaissent pas entièrement, mais ils sont souvent expurgés ou adaptés. La plupart de leurs aspects irrationnels (tels qu'un loup habillé et qui parle) sont supprimés et les moralités soulignées.

\section{Geschiedenis van Rood-Kapje - Histoire du Petit Chaperon-Rouge, $\mathrm{N}^{\circ}$ 220, Turnhout, Brepols \& Dierckx zoon, I880-I9II}

On s'attendrait à ce que la dernière planche (fig. 3), dont une première édition semble être publiée à Turnhout en Belgique, par Brépols \& Dierckx zoon, après I880, suive cette tendance et propose à ses jeunes lecteurs une version du conte dans laquelle les scènes violentes ou sexuelles aient été omises ou soient du moins atténuées. Or cette troisième version du «Petit Chaperon rouge» est de loin la plus effrayante. Cet effet est dû au choix des scènes et à leur disposition sur la feuille, mais aussi à plusieurs innovations iconographiques du créateur. Nous commencerons notre analyse par le premier aspect, qui indique déjà l'objectif de l'adaptateur, avant de développer plus amplement les éléments novateurs des illustrations qui soulignent le caractère angoissant de cette planche.

La feuille se compose de vingt illustrations réparties sur quatre bandes (A à $\mathrm{D})$ de cinq images, accompagnées de légendes bilingues de quatre lignes. On retrouve donc la structure linéaire traditionnelle mais, comme nous le verrons plus bas, l'adaptateur a clairement tiré profit des rapprochements verticaux que le lecteur peut faire pendant sa lecture et les effets qu'il a ainsi créés sont riches de sens. L'effet angoissant de cette planche est dû, d'abord, au fait que l'adaptateur a mis l'accent sur la seconde partie de l'histoire. Il a réduit le nombre d'images consacrées à l'exposition et à l'intrigue. 


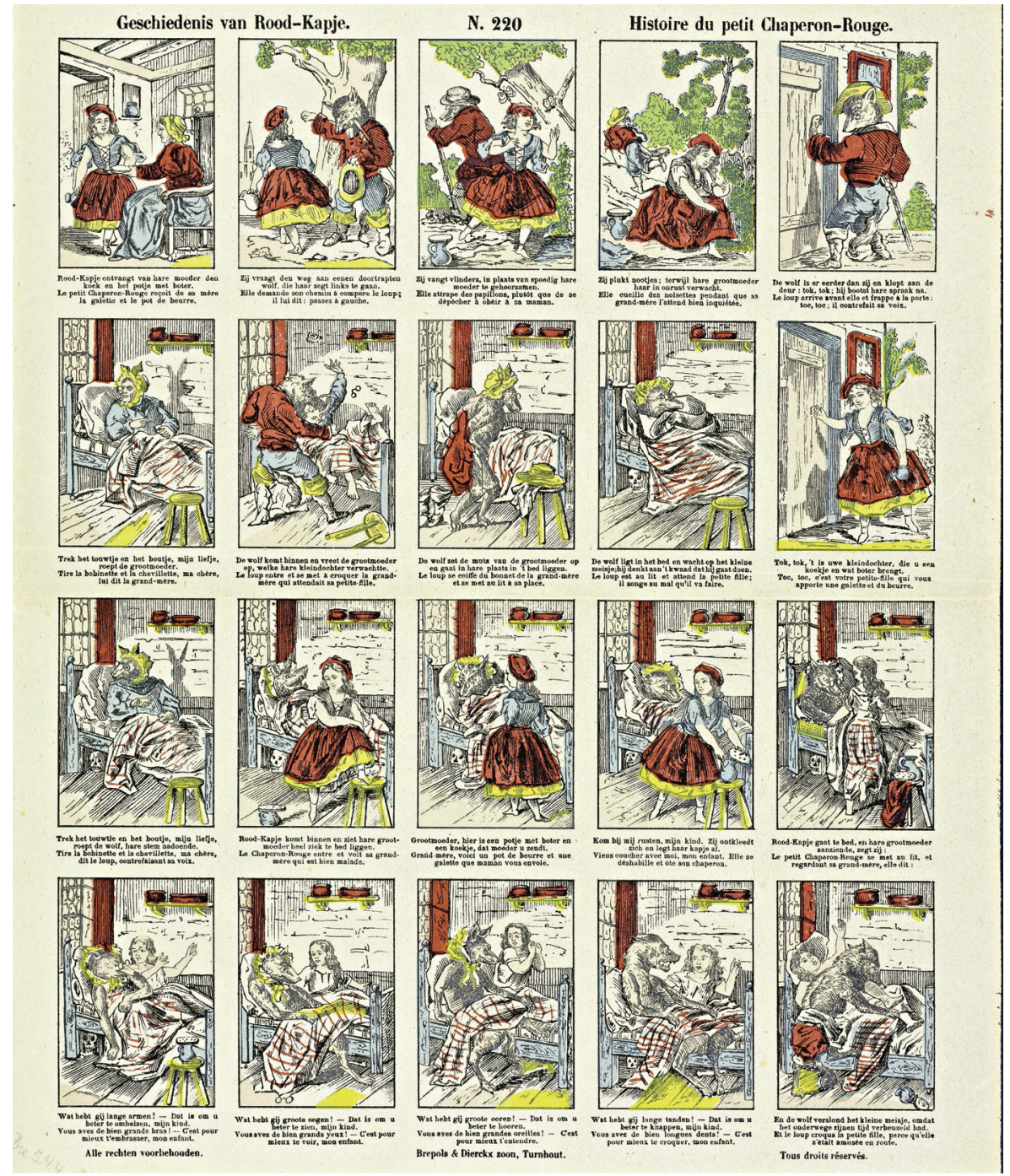

Fig. 3. - Geschiedenis van Rood-Kapje - Histoire du petit Chaperon Rouge.

N. 220, Turnhout, Brepols \& Dierckx zoon, I880-19II. 20 gravures sur bois de bout coloriées au pochoir en rouge, jaune et bleu clair, $37 \times 3 \mathrm{I} \mathrm{cm}$. Bibliothèque royale (KB), La Haye : Borms 0285 .

La première illustration (AI) permet d'introduire l'héroïne. Bien que la galette et le pot de beurre aient été représentés dans plusieurs illus- 
trations, la mission de l'héroïne, déjà connue du public, reste implicite dans le texte. L'auteur précise uniquement dans la légende que la jeune fille reçoit ces deux objets de sa mère. L'intrigue correspond à la seconde illustration (A2). Le Chaperon fait la rencontre du loup au bord de la forêt et lui demande son chemin. On remarque que dans cette version c'est la fille qui prend l'initiative de la conversation avec le loup, alors que dans les planches précédentes c'était le loup qui l'abordait. Les huit images $\left(\mathrm{A}_{3}-\mathrm{B}_{5}\right)$ qui suivent correspondent au climax, retraçant le parcours de l'hérö̈ne à travers la forêt, l'arrivée du loup chez la grand-mère, la première dévoration et enfin le moment où la fillette frappe à la porte. La catastrophe et le dénouement occupent les deux dernières lignes ( $\left.\mathrm{C}_{-}-\mathrm{D}_{5}\right)$. On remarque donc que deux images seulement ont été consacrées à l'exposition et l'intrigue tandis que le climax, la catastrophe et le dénouement en occupent dix-huit. Ainsi l'adaptateur met l'accent sur la seconde partie de l'histoire, et par conséquent sur ses aspects violents et sexuels.

En outre, cette disposition remarquable permet au créateur de la planche de jouer sur le rythme de la narration. Dans un premier temps l'histoire semble s'accélérer. L'adaptateur passe rapidement sur l'exposition et l'intrigue, ce qui donne l'impression que la jeune fille s'approche de plus en plus rapidement de son horrible sort. Cet effet est renforcé par le nombre plus élevé d'illustrations par bande et par les espaces blancs relativement étroits entre elles. Or pendant la seconde moitié de l'histoire, le rythme du récit ralentit. L'adaptateur insiste sur la dévoration de la grand-mère, et surtout sur le dialogue entre le loup et la jeune fille. Pour souligner le caractère angoissant de ces passages, l'adaptateur a ajouté plusieurs illustrations dont la composition est presque identique. Il s'agit de deux séries (BI-B4 et $\mathrm{CI}_{-}-\mathrm{D}_{5}$ ) représentant successivement la dévoration de la grandmère et de la jeune fille, et de quatre illustrations, à savoir $\mathrm{A}_{5}$ et $\mathrm{B}_{5}$, figurant l'arrivée du loup et du Chaperon chez la grand-mère, et Bi et Ci donnant à voir la grand-mère et le loup au lit. Il invite le lecteur à apprécier l'effet presque cinématographique des séries. Les quatre illustrations soulignent visuellement les répétitions typiques du conte. Plusieurs innovations iconographiques, notamment dans cette seconde partie, viennent renforcer le caractère inquiétant de cette planche.

Premièrement, on constate que l'adaptateur a limité la présence humaine dans les illustrations de cette planche. Trois images seulement (AI, BI et B2) représentent d'autres personnages, à savoir la mère et la grand-mère. Dans les versions précédentes la tension dramatique est réduite par la présence de maisons (fig. I : AI, BI, CI ; fig. 2 : I, 2), de moulins (fig. I : B3) ou de bûcherons (fig. I : B2 ; fig. 2 : II, I). Ces éléments, suggérant une 
issue de secours pour la jeune héroïne, ont complètement disparu dans la troisième planche. Par conséquent, le Petit Chaperon rouge se trouve presque toujours en tête-à-tête avec le loup. Il n'y a qu'une seule exception, qui devient d'autant plus significative : dans la seconde image (A2), lorsque le loup lui dit d'aller à gauche, ce dernier indique en même temps une église qui se trouve à l'horizon, comme pour signifier que Dieu seul pourra la sauver.

Deuxièmement, le rapprochement visuel du loup et de la jeune fille est souligné par le nombre nettement plus élevé d'illustrations dans lesquelles ces deux protagonistes sont représentés ensemble, ainsi que par le manque d'horizon. Dans la troisième planche, douze illustrations sur vingt représentent le Chaperon et le loup, alors que dans les deux planches précédentes il n'y en avait respectivement que cinq sur douze et trois sur quatorze. De plus, les personnages se trouvent presque toujours très proches l'un de l'autre dans un espace restreint. Dans la forêt, ils se trouvent entourés d'arbres et de buissons qui cachent l'horizon, et à partir de l'image Bi l'histoire se déroule presque exclusivement à l'intérieur de la maison de la grand-mère, dont on ne voit même pas la chambre entière. À gauche, on remarque une partie d'une fenêtre, mais contrairement à la porte ouverte de l'image AI, cette fenêtre reste fermée et on ne peut pas voir le paysage à l'extérieur. Dans les toutes dernières images (Di-D5), l'adaptateur semble créer un effet de zoom sur le lit qui devient une sorte de scène miniature à l'intérieur de l'image, sur laquelle se joue l'action : les parties visibles de la fenêtre et du plancher sont réduites et le tabouret disparait, par conséquent le spectateur semble s'approcher encore plus du lit.

Troisièmement, l'adaptateur a mis l'accent sur l'imprudence voire la frivolité de la jeune fille. Non seulement c'est elle qui prend l'initiative de la conversation avec le loup, mais l'adaptateur la représente en train de courir après les papillons $\left(A_{3}\right)$ et de cueillir des noisettes ( $\left.A_{4}\right)$, alors que dans les planches précédentes une image seulement était consacrée à ce passage du conte (fig. I : $\mathrm{CI}$; fig. 2 : II, 4 ). Les légendes de ces deux images rendent explicite que la jeune héroïne commet une erreur : elle préfère s'amuser au lieu d'obéir à sa mère et elle ne pense pas à sa grandmère qui l'attend et s'inquiète pour elle. Enfin, on remarque aussi que le Petit Chaperon rouge n'est plus une enfant sage, comme dans la planche de Noothoven. Comme l'indique sa différence de taille avec sa mère et le loup, l'adaptateur la représente plutôt comme une jeune fille. Elle est peut-être même un peu coquette, comme le suggèrent ses longs cheveux bouclés dénoués et ses gestes élégants lorsqu' elle enlève ses bottes et se déshabille $\left(\mathrm{C}_{2}-\mathrm{C}_{5}\right)$. 
Quant au loup, l'adaptateur souligne son caractère rusé et sa violence dès le début de l'histoire. Dans la légende néerlandaise de l'image A2, il introduit le compère loup sans aucune équivoque comme "un loup très méchant et rusé» ("eenen doortrapten wolf»). Le loup contrefait la voix de la jeune fille lorsqu'il frappe à la porte de la grand-mère $\left(A_{5}\right)$ et celle de la grand-mère lorsque le Chaperon arrive $(\mathrm{CI})$, un élément omis dans le texte des deux autres planches. Lorsque le loup dévore la grand-mère, la violence de la scène est soulignée par les gestes désespérés de la grandmère, par ses lunettes et une petite boîte lancées en l'air, et par le tabouret renversé (B2). Cette scène est d'autant plus cruelle que la grand-mère était représentée dans l'image précédente comme une bonne petite vieille qui appelle sa petite fille "ma chère" ("mijn lieffe») et qui ne se doute pas de la ruse du loup. L'adaptateur semble avoir voulu souligner le contraste entre ces deux personnages en rendant «visible» le bon caractère de la grand-mère. Comme elle porte un bonnet de nuit avec un grand ruban, son ombre projetée au mur derrière elle se transforme en lièvre ou en biche, petit animal innocent qui va devenir la proie du méchant loup (BI). Ce dernier efface tous les signes de violence. Il remet debout le tabouret et cache sous le lit les vêtements et le crâne de la grand-mère (!), figuré par une tête de mort. Il enfile ensuite la chemise de nuit de cette dernière, chausse ses lunettes et met son bonnet. L'image CI, placée très significativement en dessous de celle représentant la grand-mère au lit, montre à quel point l'illusion est parfaite : même l'ombre du loup se transforme en petit lapin! Aussi l'héroïne n'hésite-t-elle pas à s'approcher du lit.

Dans la dernière partie de l'histoire, l'adaptateur insiste encore sur les connotations sexuelles et la violence du conte. En effet, dans cette planche, le loup est presque toujours représenté comme un homme. Il est habillé, il marche sur ses deux pattes de derrière, il "parle» (voir les légendes de huit images : $\mathrm{A}_{2}, \mathrm{~A}_{5}, \mathrm{C}_{1}, \mathrm{C}_{4}$, Di-D4) et cinq images représentent les deux protagonistes au lit. De plus, l'adaptateur augmente le suspense en multipliant les scènes retardant la dévoration finale. Les images $\mathrm{C}_{2}$ à $\mathrm{C}_{5}$ représentent le déshabillage de l'héroïne. Puis les images Di à Ds reproduisent le dialogue entre le loup et le Chaperon presque entièrement, tandis que d'habitude ce dialogue est réduit à la dernière question menant à la dévoration de l'héroïne. Une ou deux images seulement évoquent alors cet épisode (fig. I : $\mathrm{D}_{2}, \mathrm{D}_{3}$; fig. 2 : III, 4). Dans la troisième planche, ces neuf images dont la composition est presque identique, permettent de créer un effet presque cinématographique. D'une image à l'autre, l'illusion se brise et le loup montre un peu plus sa vraie nature. Tandis qu'il est d'abord couché et caché sous les draps, les questions de la jeune fille 
vont lui permettre de dévoiler d'abord ses grands bras (Di), ses yeux ( 2 2; les lunettes tombent alors par terre), ses oreilles $\left(\mathrm{D}_{3}\right)$ et ses dents ( 44$)$. À chaque fois son attitude devient plus menaçante, et le regard du Chaperon plus anxieux. Dans les deux dernières images, le loup perd le bonnet de la grand-mère et redevient un animal sauvage qui finit par dévorer l'hérö̈ne. La tête de mort et parfois quelques os restent visibles sous le lit dans la plupart des images et annoncent le sort horrible et implacable de la jeune fille. La légende de la dernière image (D5) précise encore qu'elle l'a pourtant mérité : le loup la dévore parce qu'elle a perdu son temps en route ("omdat het onderweegen zijnen tijd verbeuzeld had»). La galette et le pot de beurre se trouvent par terre au pied du lit comme pour souligner l'échec de la mission de la jeune fille.

$\mathrm{Au} \mathrm{XIX}^{\mathrm{e}}$ siècle les contes sont toujours sujets à débat. Certains continuent à les considérer comme de mauvaises lectures pour les enfants. Cependant une nouvelle vision émerge, qui annonce la réhabilitation de la fantaisie pendant la période romantique. Nicolaas Beets (I8I4-I9O3), un auteur néerlandais extrêmement populaire qui écrit sous le pseudonyme Hildebrand et dont le roman Camera Obscura (I854) et les poésies religieuses sont encore célèbres aujourd'hui, plaide pour les contes de fées. Dans son article «Vooruitgang» (Progrès), publié d'abord séparément en I837 dans le journal littéraire De Gids (Le Guide) avant d'être ajouté à Camera Obscura, il en valorise notamment les aspects effrayants et merveilleux :

Et pourtant je crois que fermer entièrement les enfants au monde surnaturel et limiter leur univers au domaine du physiquement possible a un côté fâcheux et crée, dans mainte âme juvénile, une certaine prédisposition au scepticisme et au rationalisme, ou du moins une sorte d'insensibilité à bien des choses qui auraient autrement ému leur cœur. En effet, on prive la jeunesse de trop d'impressions. Nos petits bonhommes sont trop raisonnables, trop sages. [...] Une âme d'enfant veut avoir ses horreurs. Que le merveilleux est tentant! D'ailleurs, ne lisez-vous pas des histoires de fantômes ou de merveilles avec plaisir ${ }^{17}$ !

17. "En toch geloof $i k$, dat het geheel afsluiten dier bovennatuurlijke wereld, het volstrekt beper ken der kinderlijke begrippen tot het gebied van het fysiek-mogelijke, zijne, kwade zijde heeft, en in menig jeugdige ziel den grond legt tot een later scepticisme, rationalisme of ten minste tot een zekere koelheid voor een menigte zaken, die anders op het gemoed plegen te werken. Waarlijk, men maakt der jeugd te veel indrukken onmogelijk. Onze kleine mannetjes zijn al te verstandig, al te wijs. [...] Eene kinderziel wil hare verschrikkingen hebben. Het wonderbaarlijke - hoe verlokkelijk is het! Of is het uzelven niet een genoegen spook en wondergeschiedenissen te lezen!» Hildebrand (Nicolaas Beets), Vooruitgang, Camera Obscura, 1917, p. 275. [Nous traduisons.] 
La planche de Brepols \& Dierckx zoon, dans laquelle les aspects angoissants et merveilleux du conte «Le Petit Chaperon rouge» sont renforcés, semble refléter cette vision.

\section{Conclusion}

L'analyse des trois planches populaires néerlandaises représentant «Le Petit Chaperon rouge» montre que même si, à première vue, elles se ressemblent beaucoup, elles présentent néanmoins des différences notables. D'une planche à l'autre, le nombre d'images et leur disposition sur la feuille, le sens de la "lecture», la technique d'illustration, la composition, le choix des scènes, enfin, varient sensiblement. Les adaptateurs créent ainsi différentes versions d'un même conte, qui semblent refléter différentes opinions ayant cours dans les débats pédagogiques, et qui permettent de retracer l'évolution du livre pour enfants.

Létude des planches offre également des informations sur les méthodes de travail des éditeurs. La planche de Erve $\mathrm{H}$. Rynders a été rééditée jusqu'à la fin du XIX ${ }^{e}$ siècle. Au moins cinq éditions presque identiques ont survécu, indice clair du succès de cette version. Les éditeurs, pour continuer à la publier, sont même prêts à en faire graver des copies au cas où ils ne disposent pas des gravures originales. Ce travail à la fois précis et coûteux semble en contradiction avec leurs pratiques souvent nonchalantes et le prix modeste des planches. La version du conte proposée par cette planche, plus proche de celle de Perrault, était peut-être davantage appréciée du public que les nouvelles planches publiées par Noothoven van Goor et Brepols \& Dierckx zoon, dont on ne trouve que très peu de rééditions ${ }^{18}$. Il est intéressant de remarquer qu'apparemment plusieurs planches différentes figurant «Le Petit Chaperon rouge» étaient disponibles en même temps, aussi le lecteur pouvait-il choisir la version qui lui plaisait le plus ou qui correspondait le mieux à ses conceptions éducatives. Les planches

I8. Deux versions existent de la planche de Noothoven van Goor, portant les numéros 204 et $2 \mathrm{I} 2$ du catalogue des publications de la maison. À notre connaissance la planche n’a pas été (ré)éditée par d'autres éditeurs. Nous n'avons trouvé que trois exemplaires de la troisième planche. Outre l'édition de Brepols \& Dierckx zoon (Geschiedenis van Rood-kapje - Histoire du Petit Chaperon rouge, № 220, Turnhout, Brepols \& Dierckx zoon, I880-I9II), existent une édition anonyme, intitulée également Geschiedenis van Rood-kapje - Histoire du Petit Chaperon rouge, № 220 (I800-1900) (Sneek, Fries Scheepvaart Museum, E-309) et une réédition des Établissements Brepols S.A. (Turnhout, I9II-1935) (La Haye, Koninklijke Bibliotheek, Borms 0284). 
reflètent ainsi non seulement l'évolution des théories pédagogiques, mais elles témoignent également du fait qu'au XIX ${ }^{e}$ siècle, l'offre d'ouvrages pour les enfants gagne en variété : c'est la preuve que la littérature de jeunesse est désormais une branche à part entière de la librairie. Les illustrations jouent un rôle central dans ce processus de renouvellement et de diversification.

\section{Bibliographie SÉLECTIVE}

Borms Aernout, "Centsprenten: de cartoons van weleer", dans Origine, tijdschrift voor kunst, antiek en toegepaste kunst, vol. I4, $\mathrm{n}^{\circ}$ 2, 2006, p. 50-56, <www.hetoudekinderboek.nl/Centsprenten/ ProjectCentsprenten/InleidingCentsprenten.htm> (consulté le Is avril 20I4).

Buijnsters P. J. et Bujunsters-Smets Leontine, Lusten Leering. Geschiedenis van het Nederlandse kinderboek in de negentiende eeuw, Zwolle, Waanders, 200 .

Delft Marieke van (éd.), Wonderland. De wereld van het kinderboek, Zwolle, Waanders, 2003.

Dongelmans Berry (éd.), Tot volle waschdom. Bijdragen aan de geschiedenis van de kinder-en jeugdliteratuur, La Haye, Biblion, 2000.

Gaillard Aurélia, Fables, Mythes, Contes. L'esthétique de la fable et du fabuleux (I660-I729), Paris, H. Champion, 1996.

Gourevitch Jean-Paul, Images d'enfance. Quatre siècles d'illustration du livre pour enfants, Paris, Éditions Alternatives, 1994.

Hildebrand (Nicolaas Beets), Vooruitgang, Camera Obscura, Haarlem, De Erven F. Bohn, 1917, p. 273-279. Project Gutenberg: <www. gutenberg.org/files/15975/15975-h/I595-h.ht - xdoeI3748> (consulté le I5 avril 20I4).

Hoogenboezem Daphne M., Le conte de fées en images. Le rôle de l'illustration chez Perrault et Madame d'Aulnoy (I695-I800), thèse doctorale, Université de Groningen, Pays-Bas, 2012.

Le Men Ségolène et Renonciat Annie (éd.), Livres d'enfants, livres d'images (I848-I9I4), Les Dossiers du musée d'Orsay, $\mathrm{n}^{\circ}$ 35, Paris, Réunion des musées nationaux, 1989 .

Nières Isabelle, "Une planche d'Épinal : Histoire de la Barbe-Bleue : NE IIO2", dans J. Perrot (éd.), Tricentenaire Charles Perrault - Les grands contes du XVII siècle et leur fortune littéraire, Paris, In-Press, I998, p. $75-84$. 
Perrault Charles, Contes, J.-P. Collinet (éd.), Paris, Gallimard, I98I.

Perrot Jean (éd.), Tricentenaire Charles Perrault - Les grands contes $d u$ $X V I I^{e}$ siècle et leur fortune littéraire, Paris, In-Press, 1998.

Picaud Carine, "L'illustration du conte de fées. Enjeux d'images, visions d'imagiers", dans O. Piffault (éd.), Il était une fois... les contes des fées, Paris, Seuil, 200I.

Piffault Olivier (éd.), Il était une fois... les contes des fées, Paris, Seuil, 200I.

RAYNARD Sophie, "Perrault et les conteuses précieuses de la génération I690: Dialogue ou querelle masquée", The Romanic Review, vol. 3, $\mathrm{n}^{\mathrm{o}} 4$, 2008, p. 317-33I.

Renonciat Annie, "Le Petit Poucet en images", dans S. Le Men et A. Renonciat (éd.), Livres d'enfants, livres d'images (I848-I9I4), Les Dossiers du musée d'Orsay, $\mathrm{n}^{\circ}$ 35, Paris, Réunion des musées nationaux, 1989.

—, "Petit Poucet dans la jonchée de feuilles", Le Vieux Papier, no 316, I990, p. 205-2I8.

Van Effen Justus, Le Misanthrope (tome 3), Amsterdam, I745.

Wolff-Bekker Elizabeth, Proeve over de opvoeding aan de Nederlandse moeders, Amsterdam, Johannes Allart, I780. 